\title{
Regulators weigh benefits of 'three-parent' fertilization
}

\section{But critics say mitochondrial replacement carries safety and ethical concerns.}

\section{BY ERIKA CHECK HAYDEN}

$\mathrm{R}$ egulators in the United States are considering whether to permit trials of a controversial assisted-reproduction technique intended to help women to avoid passing certain genetic defects on to their children.

On 22 October, the US Food and Drug Administration (FDA) is scheduled to meet in Silver Spring, Maryland, to discuss a method that could prevent transmission of defects in mitochondria - cellular components that contain a small amount of DNA - from mother to child. The defects, which can cause fatal developmental conditions, affect as many as 4,000 US births a year.

The technique places nuclear DNA from the egg of a woman with a mitochondrial defect into a donated egg that has had its nuclear DNA removed, but contains healthy mitochondrial DNA. Once the egg is fertilized, the resulting embryo would, in a sense, have three parents, because the donor mitochondrial DNA is passed down along with the mother and father's nuclear DNA.

The FDA was asked to look into the issue by developmental biologist Shoukhrat Mitalipov at Oregon Health and Science University in Beaverton, who last year created early human embryos with the technique (see Nature http:// doi.org/n76; 2012). When the manipulated eggs were fertilized, genetic abnormalities were detected in half of them - but seemingly normal embryonic stem-cell lines could be extracted from $38 \%$ of the rest. Trying to obtain stem cells from unmanipulated eggs results in a similar success rate. Mitalipov had used the same technique in 2009 to create apparently healthy rhesus monkeys. Now he wants to begin a clinical trial in humans.

In 2001, the FDA began to regulate the technique as a form of gene therapy after researchers used fresh mitochondria in a handful of infertile women to help them to conceive (see 'Energizing eggs'). The regulation was widely, but incorrectly, reported as a ban. The FDA asked researchers to apply for permission to test the approach in clinical trials. But none did - until now. At the time, the agency said that the safety data "were not convincing", citing examples of genetic abnormalities such as a missing $\mathrm{X}$ chromosome

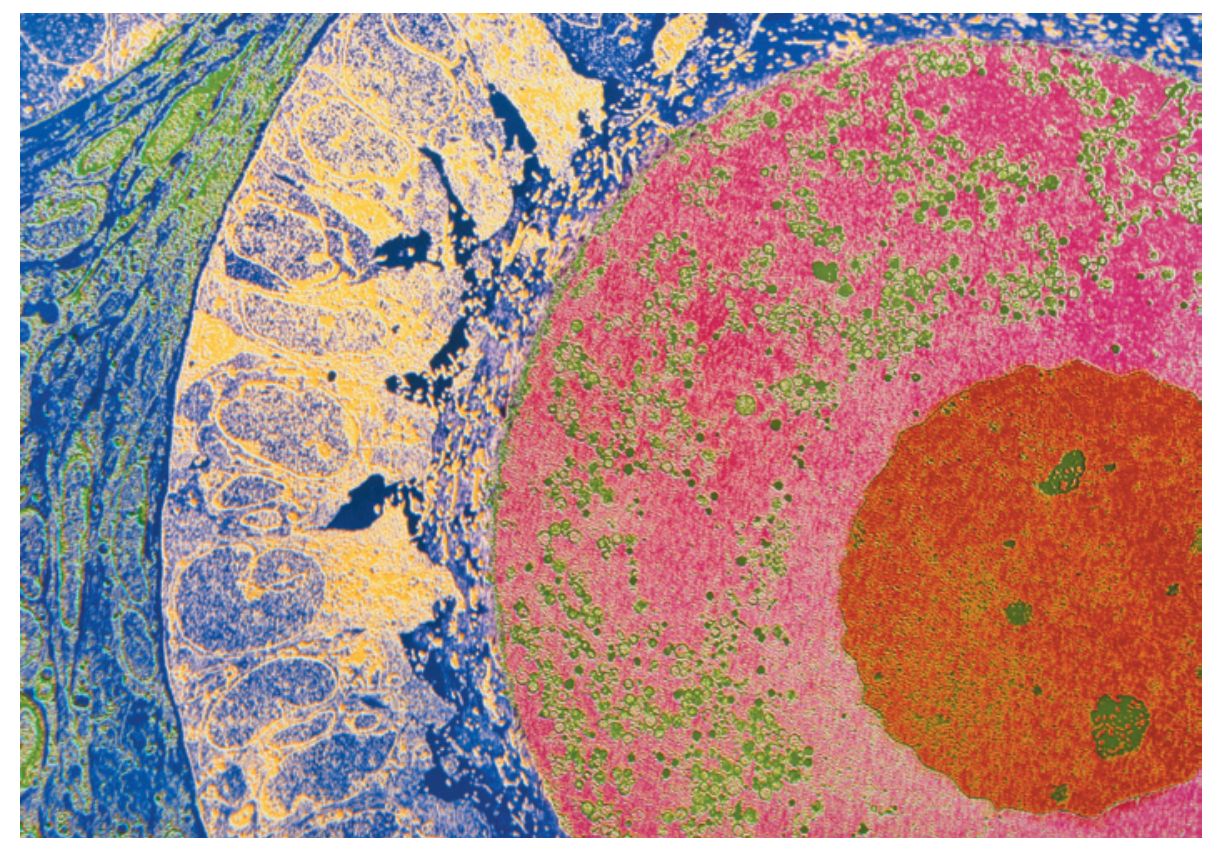

Mitochondria (green) in egg cells carry an independent lineage of DNA that can pass on genetic defects.

in a fetus created with the technique.

The anomalies seen in embryos created with mitochondrial transfer could have been due to the mothers' underlying fertility issues rather than to the technique itself, says embryologist Jacques Cohen, who was scientific director of assisted reproduction at Saint Barnabas Medical Center in Livingston, New Jersey, when such treatments were conducted there.

But other safety concerns have been raised since then. In September, a group of evolutionary biologists led by Klaus Reinhardt at the University of Tübingen in Germany, said that problems could arise if mitochondrial and nuclear DNA from different women proved to be incompatible. They pointed to dozens of experiments in mice, fruit flies and other animals in which mixing nuclear and mitochondrial DNA from individuals with different genetic backgrounds sometimes led to reduced growth, early death, fast ageing or reduced reproductive ability.

\section{$\rightarrow$ NATURE.COM}

For more on mitochondrial replacement, see: go.nature.com/xhdcdw
Mitalipov and other scientists counter that those experiments were mostly done by mixing strains of inbred animals. In species such as humans, individuals from different genetic backgrounds interbreed freely without ill effects. "If anything, children born from mixed-race couples, and [their] successive generations, are fitter than those from same-race couples," says developmental geneticist Robin Lovell-Badge of the Medical Research Council National Institute for Medical Research in London.

Paul Knoepfler, a stem-cell biologist at the University of California, Davis, has a different concern: epigenetics. He says that the donor egg's cytoplasm could reprogram chemical tags on the nuclear DNA which alter the expression of genes. But Mitalipov argues that reprogramming will not occur with his technique because he is transferring genetic material between cells that are in exactly the same developmental state. He points to the existence of the healthy monkeys that are now more than four years old - and are the product of mitochondrial transplants across different genetic backgrounds - as evidence that the technique is safe.

In March, the UK Human Fertilisation and Embryology Authority (HFEA) concluded that human trials could be done if, for instance, offspring were monitored long-term. The UK government is now drawing up regulations 


\section{ENERGIZING EGGS}

\section{Experimental fertility treatment faces scrutiny}

As the US Food and Drug Administration (FDA) debates the merits of mitochondrial replacement in eggs, some observers will be looking for hints as to how the agency may regulate another mitochondrial manipulation - one with fewer ethical and safety concerns.

OvaScience, a biotechnology company in Cambridge, Massachusetts, wants to boost the success rate of in vitro fertilization (IVF) by infusing eggs with fresh mitochondria. The mitochondria are harvested from an IVF patient's own egg precursor cells, a cell type discovered by Jonathan Tilly, a reproductive biologist at Northeastern University in Boston, Massachusetts. Tilly says that these precursor cells can be coaxed to develop into mature eggs in adult women, challenging the dogma that women are born with all the eggs they will ever have. Tilly's results are disputed (see Nature 491, 318-320; 2012), but OvaScience has longterm plans to harvest precursor cells and use them to create fresh eggs for women for whom conventional IVF has failed.

The company's first project, called AUGMENT, is to harvest precursor cells, isolate their mitochondria, and inject

\section{EGG REGS}

OvaScience shares plummeted on 10 September, the day the company revealed that the US Food and Drug Administration might regulate its fertility treatment.

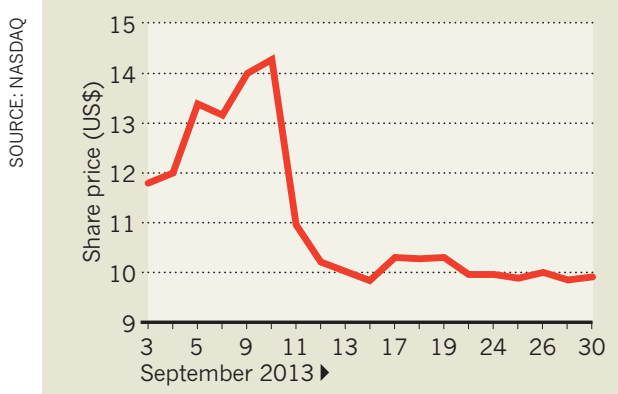

on the technique, and Parliament, which had banned all germline modifications, will vote on whether to allow the procedure next year.

There are also ethical considerations. The HFEA said that the procedure should be considered in the same ways as a tissue donation, and that any resulting child should not have the right to know the identity of the donor of the healthy mitochondria. The FDA, unlike the HFEA, does not consider ethics, and that worries Marcy Darnovsky, executive director of the Center for Genetics and Society, an advocacy group in Berkeley, California. Her group has opposed such trials, in part because of concerns that acceptance of the technology might lead to the selection of embryos with them into mature eggs to see if they can revive eggs from infertile women, as work with mitochondria from donor eggs has suggested. Mitochondrial DNA from eggprecursor cells is thought to contain fewer mutations than mitochondrial DNA in the eggs themselves. Because OvaScience would be using mitochondria from a patient's own cells, the company hopes to sidestep ethical concerns raised by 'three-parent' embryos.

OvaScience has argued that AUGMENT involves 'minimal manipulation' - the same injection procedure, for example, is already used to put sperm into an egg and therefore would not need FDA approval to be deployed in clinics. Regenerative Sciences in Broomfield, Colorado, has also argued that one of its stem-cell therapies involves 'minimal manipulation'. The FDA challenged that idea, and its injunction on the treatment was ultimately upheld in court (see Nature 488, 14; 2012).

OvaScience investors clearly feared that AUGMENT would meet the same fate when, on 10 September, the company announced that the FDA had issued a letter questioning whether the project was exempt from agency review. OvaScience voluntarily suspended enrolment in the US arm of its AUGMENT clinical study, pending a meeting with regulators. The company's shares fell $23 \%$ that day, and have yet to recover (see 'Egg regs').

But analyst Jeffrey Cohen of Ladenburg Thalmann, a financial services company in Miami, Florida, says that the FDA letter has not changed his favourable assessment of OvaScience. The AUGMENT study is continuing in Europe, he notes, where the market for IVF is as much as three times larger than in the United States, and regulatory hurdles are not expected to be a barrier. Heidi Ledford

specific traits for non-medical reasons.

Mitalipov agrees that any trial would need to proceed with caution, but says that if he cannot perform the trials in the United States, he would consider going to the United Kingdom if it allows the procedure first. "Patients are suffering the same issues, no matter where they are."

\section{CORRECTION}

The print version of the World View by George Church (Nature 502, 143; 2013) was published before the author had approved changes. The online version was amended to better reflect his views. 